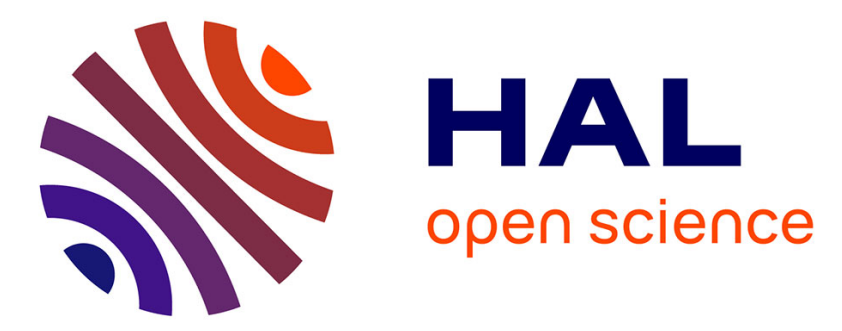

\title{
Dynamiques intergroupes et changement social autour de l'ouverture du mariage aux couples de même sexe en France
}

\author{
Anna Savio, Élise Vinet, Marie Préau
}

\section{- To cite this version:}

Anna Savio, Élise Vinet, Marie Préau. Dynamiques intergroupes et changement social autour de l'ouverture du mariage aux couples de même sexe en France. Nouvelle revue de psychosociologie, 2016, 22 (2), pp.181 - 192. 10.3917/nrp.022.0181 . halshs-01561823

\section{HAL Id: halshs-01561823 \\ https://shs.hal.science/halshs-01561823}

Submitted on 13 Jul 2017

HAL is a multi-disciplinary open access archive for the deposit and dissemination of scientific research documents, whether they are published or not. The documents may come from teaching and research institutions in France or abroad, or from public or private research centers.
L'archive ouverte pluridisciplinaire $\mathbf{H A L}$, est destinée au dépôt et à la diffusion de documents scientifiques de niveau recherche, publiés ou non, émanant des établissements d'enseignement et de recherche français ou étrangers, des laboratoires publics ou privés. 
DYNAMIQUES INTERGROUPES ET CHANGEMENT SOCIAL AUTOUR DE L'OUVERTURE DU MARIAGE AUX COUPLES DE MÊME SEXE EN FRANCE

Anna Savio, Élise Vinet, Marie Préau

ERES | « Nouvelle revue de psychosociologie »

2016/2 N²2 | pages 181 à 192

ISSN 1951-9532

ISBN 9782749252322

Article disponible en ligne à l'adresse :

http://www.cairn.info/revue-nouvelle-revue-depsychosociologie-2016-2-page-181.htm

\section{Pour citer cet article :}

Anna Savio et al., « Dynamiques intergroupes et changement social autour de l'ouverture du mariage aux couples de même sexe en France », Nouvelle revue de psychosociologie 2016/2 ( $\left.\mathrm{N}^{\circ} 22\right)$, p. 181-192.

DOI 10.3917/nrp.022.0181

Distribution électronique Cairn.info pour ERES.

(C) ERES. Tous droits réservés pour tous pays.

La reproduction ou représentation de cet article, notamment par photocopie, n'est autorisée que dans les limites des conditions générales d'utilisation du site ou, le cas échéant, des conditions générales de la licence souscrite par votre établissement. Toute autre reproduction ou représentation, en tout ou partie, sous quelque forme et de quelque manière que ce soit, est interdite sauf accord préalable et écrit de l'éditeur, en dehors des cas prévus par la législation en vigueur en France. Il est précisé que son stockage dans une base de données est également interdit. 


\title{
Dynamiques intergroupes et changement social autour de l'ouverture du mariage aux couples de même sexe en France
}

\author{
Anna Savio, Elise Vinet et Marie Préau
}

En avril 2013, après des mois de débats politiques et publics et plusieurs mobilisations collectives de grande ampleur qui ont rassemblé, de part et d'autre, aussi bien les soutiens que les opposants au projet, la société française a vu l'institution du mariage s'ouvrir aux couples de même sexe. Cet événement et le contexte social dans lequel il s'inscrit révèlent un certain nombre de dynamiques psychosociales relatives aux relations intergroupes, et notamment des enjeux spécifiques au rapport entre minorités et majorités sociales ; en effet, les populations LGBTIO $^{1}$ et en particulier homosexuelles, directement concernées par cette évolution légale, ont été particulièrement visibles et engagées. Pour approcher ce contexte social dans sa complexité, on s'intéressera notamment au changement légal en tant que voie effective vers la transformation du champ social et idéologique (Felstiner, Abel et Sarat, 1981). Dans un premier temps, nous interrogerons les enjeux des positionnements sociaux majoritaires et minoritaires, en montrant comment les minorités sexuelles peuvent se situer relativement aux normes dominantes de genre et de sexualité, et plus largement aux normes de conjugalité incarnées par l'institution du mariage. Sur ces bases et au-delà des enjeux strictement légaux, nous envisagerons ensuite, à la lumière d'une lecture et d'une interprétation psychosociale, les implications idéologiques et sociétales possibles de l'ouverture du mariage aux couples de même sexe.

Anna Savio, doctorante en psychologie sociale, laboratoire GRePS, université Lumière Lyon 2, Institut de psychologie. anna.savio@univ-lyon2.fr

Élise Vinet, maître de conférences en psychologie sociale, laboratoire GRePS, université Lumière Lyon 2, Institut de psychologie. elise.vinet@univ-lyon2.fr Marie Préau, professeure de psychologie sociale, laboratoire GRePS, université Lumière Lyon 2, Institut de psychologie. marie.preau@univ-lyon2.fr

1. LGBTIO : lesbiennes, gaies, bisexuelles, trans, intersexes, queer. 


\section{LA CATÉGORISATION SOCIALE DE SEXE : UN SYSTÈME NORMATIF}

Pour comprendre ces enjeux sociétaux, il s'agit de considérer un champ normatif majoritaire relatif au sexe, au genre et à la sexualité, qui simultanément détermine les représentations collectives, définit les identités minoritaires et constitue le fondement idéologique des institutions - et en particulier de l'institution du mariage. En effet, la plupart des systèmes d'organisation sociale accordent une importance majeure à I'assignation des acteurs à une catégorie de sexe en fonction d'un certain nombre de traits physio-biologiques (Fausto-Sterling, 2012), ces systèmes renvoyant à des normes d'autant plus injonctives qu'elles se donnent à voir comme fondées en nature (Douglas, 1999). En contexte occidental, Bem (1993) pense ce système social à partir de la notion de patriarcat, renvoyant ici à un complexe normatif fondé sur la différenciation sexuelle et l'asymétrie hiérarchique entre masculin et féminin. Socialement et structurellement incarné, celui-ci aurait tendance à s'autoréguler par le biais d'une " hétérosexualité d'institution [qui] nécessite et produit l'univocité de chaque terme » (Butler, 2005, p. 92), permettant de perpétuer la production d'une différence et d'une complémentarité symboliques entre le masculin et le féminin en tant que construits sociaux. Comme nous le verrons, cette structure normative ne concerne pas uniquement les hommes et les femmes dans une interrelation duelle. Les populations gaies et lesbiennes, et plus largement les minorités sexuelles, sont une cible privilégiée de la régulation normative, au sens où les individus persistant à être " déviants " vis-à-vis des fondements idéologiques du système s'exposent à la stigmatisation et à l'une de ses conséquences : la marginalisation sociale.

\section{L'INSTITUTION DU MARIAGE}

En tant que pilier central de ce système social, l'institution du mariage n'est pas neutre d'un point de vue normatif (Bourcier, 2010 ; Butler, 2006). En effet, au-delà de sa dimension légale et contractuelle, le mariage peut être considéré comme une structure normative, et plus concrètement comme un moyen de structurer les relations réelles et symboliques entre les individus. En France, notamment, cette institution est fortement marquée par la culture patriarcale dans laquelle elle s'inscrit (Duby et Perrot, 1992). Sur le plan social, le mariage est également indissociable des enjeux culturels liés à la procréation au sein du couple puisque son but premier est d'instituer et de réguler la filiation ; aujourd'hui encore, il est difficile de penser I'union maritale indépendamment de cet autre enjeu : la constitution d'une cellule familiale (Bologne, 1995). Au regard de son ancrage historique, le mariage reste donc une institution socialement contraignante et symboliquement marquée, structurée autour d'un ensemble de normes (telles que la monogamie, la fidélité et la filiation). Or, ces normes apparaissent comme le produit d'un ordre sexuel sousjacent qui justifie, voire affirme, la nécessité pour les partenaires d'assumer des identités et des rôles sexués différenciés dans le cadre du mariage - notamment en termes de sexualité, d'intimité et dans l'exercice de la parenté. En ce sens, " on ne peut pas se référer à l'ordre conjugal sans prendre en compte le phénomène capital auquel il renvoie : la sexualité. [...] parallèlement à l'ordre conjugal se dessine un 
ordre des sexualités qui, par le biais du mariage, place l'hétérosexualité au rang de modèle, de canon en fonction duquel toutes les sexualités doivent s'interpréter " (Borillo, 2004, p. 98). Par ailleurs, en constituant des couples légitimes par le biais de la reconnaissance légale de l'union, le mariage apparaît de fait attaché à la régulation sociale - et symbolique - de la conjugalité via une contractualisation qui en définit en les explicitant les modalités et les limites. II s'agit donc aussi d'une institution qui a le pouvoir de rendre socialement légitimes certaines formes d'union, via la reconnaissance légale. Si son ouverture aux couples de même sexe témoigne du fait que I'hétérosexualité n'est plus la condition sine qua non de cette reconnaissance légale, le mariage continue cependant de définir la notion de conjugalité et les critères de légitimité des unions.

\section{L'ORDRE SOCIAL : NORMES, REPRÉSENTATIONS ET IDÉOLOGIES}

Ces enjeux idéologiques nous invitent, à partir du concept de représentation sociale, à mobiliser la notion $d^{\prime}$ " ordre social ". En effet, alors que les représentations sociales sont pensées comme des formes de connaissances construites collectivement - et appropriées différentiellement selon les individus et les groupes - en tant que connaissance partielle de la réalité comme re-présentation, schématisation et mise en sens de celle-ci (Moscovici, 1984 ; Breakwell, 2010), I'ordre social renvoie à un ensemble de normes et de représentations articulées en système que l'on peut simultanément appréhender comme organisation concrète et comme représentation (Augé, 1974). Ici, ce sont les idéologies qui « organisent et structurent [...] le champ symbolique d'une formation sociale " (Lipiansky, 1991, p. 60), renvoyant à un " système de valeurs, de normes, de représentations, de symboles propre à une culture et une sous-culture " (Michelat cité par Jodelet, 1991, p. 17). Ajoutons que si elles façonnent le champ normatif d'une société en régulant les discours, représentations et conduites, ces idéologies sont simultanément régulées par des instances sociales telles que les institutions. Ainsi, à partir de la variabilité physiobiologique effective (génétique, hormonale, ontogénétique) liée au développement sexuel qui façonne, en relation avec les déterminants culturels, des corps sexués tendanciellement différents, des systèmes symboliques et sociaux se développent qui définissent le corps sexué comme absolument déterminant sur le plan psychique et identitaire et qui produisent dans le champ social des identités masculine et féminine idéales et idéalement hétérosexuelles, par le biais des processus d'influence sociale (Breakwell, 2010 ; Butler, 2006 ; Fausto-Sterling, 2012). Dès lors, la question du partage social des normes et représentations peut être abordée en interrogeant les positionnements individuels et collectifs relativement à un champ symbolique partagé, positionnements qui constituent et définissent des identités sociales majoritaires et minoritaires au sein de la société.

\section{CHAMP SYMBOLIQUE MAJORITAIRE ET CONSTITUTION D'IDENTITÉS MINORITAIRES}

On sait en effet que les identités individuelles et collectives, positives ou négatives, associées à nos appartenances sociales sont en partie modelées par les représenta- 
tions, normes et valeurs culturelles dominantes (Camilleri, 1990 ; Zavalloni, 2007). Or la norme - ou la représentation normative - ne peut assurer sa fonction de cohésion sociale que parce qu'elle crée le consensus à partir d'un champ symbolique spécifique et nécessairement partagé ; elle est « ce qui nous lie, mais aussi ce qui ne crée l'unité que par une stratégie d'exclusion " (Butler, 2006, p. 234). Précisément, selon la théorie de l'identité sociale (Tajfel et Turner, 1979, 1986), " I'identité est construite non pas principalement par le biais des affiliations, mais aussi par des comparaisons avec d'autres groupes grâce auxquelles une identité dominante peut émerger "(Joffe, 2004, p. 96). Les identités individuelles et collectives se constituent donc en référence au système idéologique global, mais aussi nécessairement en relation à un espace symbolique de I'Altérité, défini comme en négatif des valeurs partagées ou dominantes. En effet, un système symbolique ne peut exister que dans la mesure où il instaure son " en dehors " auquel il renvoie les éléments ne correspondant pas aux valeurs qui lui permettent de fonctionner et de se perpétuer en tant que tel (Joffe, 2004). L'identité minoritaire est donc stigmatisée à partir de " traits et stéréotypes négatifs qui font en sorte que ses possesseurs subiront une perte de statut et seront discriminés au point de faire partie d'un groupe particulier ; il y aura "eux", qui ont une mauvaise réputation, et "nous", les normaux " (Croizet, 2003, p. 14). Précisément, les critères pertinents pour penser une minorité sociale sont, d'une part, l'assignation à un statut minoritaire - sur la base de relations sociales asymétriques légitimées structurellement - et, d'autre part, " le sentiment d'appartenance au groupe, le principe d'identité posé par les individus postulant à l'existence de ce groupe " (Taboada-Leonetti, 1990, p. 59). Or, c'est sur ce sentiment d'appartenance que les minorités vont pouvoir s'appuyer pour éventuellement transformer le stigmate et revaloriser l'identité collective (Tajfel et Turner, 1979, 1986) ; il s'agit alors de passer d'une identité fondée sur des "catégories déviantes " au travers desquelles les personnes minorisées " étaient définies et se définissaient [...] de manière négative et pathologique par rapport au code social dominant " à l'investissement d'un groupe qui possède un code propre et qui vise à le proposer au reste de la société " à titre de modèle ou de solution de rechange " (Moscovici, 1979, p. 11). Ces minorités sont alors dites " nomiques " au sens où elles se définissent autour d'un code commun et de normes partagées, valorisées et reconnues au sein du groupe, distinctes de celles de la majorité sociale. On est également renvoyé ici au processus de retournement du stigmate, propre aux stratégies de créativité sociale, qui s'appuie sur une re-signification des valeurs et des normes majoritaires à l'origine de la stigmatisation, permettant une revalorisation de l'identité collective (Tajfel et Turner, 1986).

\section{DE L'IDENTITÉ STIGMATISÉE À LA MINORITÉ NOMIQUE : LE CAS DES POPULATIONS LGBTIQ}

L'histoire sociale et politique des minorités sexuelles nous permet d'illustrer avec pertinence ces processus en montrant comment une population minoritaire peut être amenée à se constituer comme groupe(s) sur la base d'une marginalisation commune. En effet, au regard des critères proposés plus haut, on peut considérer 
les populations LGBTIQ comme un groupe minoritaire - bien que composite. Les identités que rassemble l'acronyme se sont en effet développées à partir d'un stigmate relatif aux normes sexuelles, qu'elles concernent le désir (attendu comme hétérosexuel), l'identité (attendue comme féminine ou masculine en adéquation avec le sexe) ou le corps sexué (attendu comme exclusivement mâle ou femelle selon les critères culturels au fondement de la construction des catégories de sexe). Or, au fil de I'histoire sociale et politique de ces groupes a émergé la possibilité d'une coalition des minorités sexuelles autour d'un certain nombre de valeurs qui, au-delà du stigmate, les ont progressivement constituées comme sujets politiques (Fortin, 2010). Dans ce cas précis, on pense notamment au processus de recomposition identitaire, permettant la création d'une nouvelle puissance d'agir collective issue du rassemblement de différentes minorités en une entité unique et plus englobante (Taboada-Leonetti, 1990). En ce sens, on s'intéresse à des groupes qui tendent à transformer leur statut de minorités stigmatisées pour émerger dans le champ social comme minorités nomiques et potentiels vecteurs de changement social (Moscovici, 1979). En considérant les conditions et les enjeux de ce changement social, on verra que le potentiel spécifique aux minorités LGBTIO prend un sens particulier dans le contexte de l'ouverture de l'institution du mariage aux couples de même sexe.

\section{LES POLITIQUES LGBTIO : QUELLES ORIENTATIONS ?}

En effet, certaines prises de position politiques des groupes LGBTIO constituent une critique explicite des normes régulatrices appliquées au sexe, au genre et à la sexualité - incarnées par l'institution du mariage - et visent à leur transformation. En ce qui concerne la reconnaissance institutionnelle et les modalités par lesquelles une sexualité légitime est reconnue, et par là définie, on constate des divergences au sein des populations et groupes LGBTIQ, renvoyant a priori aux stratégies identitaires de similarisation ou de différenciation relativement aux normes majoritaires (Kastersztein, 1990). Concrètement, deux tendances claires se dessinent qui renvoient directement aux enjeux historiques, sociaux et politiques des minorités sexuelles (Fortin, 2010 ; Prearo, 2014). D'un côté, cette histoire groupale témoigne d'une recherche de reconnaissance de l'identité et de la sexualité selon les cadres majoritaires en vigueur, se traduisant par une volonté d'intégrer pleinement les structures existantes. Appliquée à la question du mariage et concernant le rapport du groupe aux normes majoritaires, cette recherche peut à l'extrême être considérée comme " un désir de se fondre dans la normalité, de revendiquer son in-différence, voire d'imiter le couple et la famille hétérosexuelle " (Bourcier, 2010, p. 8). Simultanément, au sein des mouvements LGBTIO, des critiques sont portées non seulement sur le mariage et la « répartition des rôles de genre qu'il suppose " (ibid., p. 9), mais aussi sur les normes de sexualité et de conjugalité telles qu'elles sont incarnées par l'institution du mariage via les principes de monogamie, de fidélité et de filiation, et ce relativement au développement dans les cultures queer de formes alternatives de sexualité et d'intimité (Warner, 1999). Bien qu'apparemment spécifiques à un groupe et à ses intérêts particuliers dans une situation donnée, ces deux tendances politiques semblent pourtant renvoyer 
plus largement à deux orientations différentes en termes de changement social. En effet, on distingue deux types de mouvements sociaux (Aberle, 1966) qui poursuivent un changement social " par une modification soit limitée (mouvements réformistes), soit profonde (mouvements transformateurs) de la structure sociale " (Fillieule, 2009, p. 22). Dans cette perspective, les revendications visant l'obtention de l'égalité des droits concernant le mariage et la filiation nous renverraient à une orientation réformiste, dont le but est de faire évoluer les modalités d'accès à l'institution pour les rendre plus inclusives, sans nécessairement questionner les dimensions idéologiques sous-jacentes à la structuration sociale à laquelle participent les institutions. D'autre part, les revendications visant un changement plus profond de ces valeurs - comme la remise en question des présupposés naturalistes sur les liens entre sexe, genre et sexualité que soutient historiquement l'institution du mariage - dessineraient une tendance politique transformatrice incarnée par une partie des communautés politiques LGBTIO. Or, ainsi qu'on va le voir, les stratégies identitaires de similarisation et de différenciation, et plus largement la conformité et l'innovation, sont des mécanismes fondamentaux du processus de transformation des normes à l'origine du changement social.

\section{CHANGEMENT SOCIAL : LE POTENTIEL SPÉCIFIOUE DES GROUPES MINORITAIRES}

L'ordre social émerge en effet comme la matérialisation de l'image consensuelle d'une société, c'est-à-dire de sa représentation telle qu'elle est partagée par les acteurs qui font cette société. Cette matérialisation, " si elle perd de son emprise sur les esprits d'une majorité suffisante ou d'une minorité avec la puissance suffisante de l'imposer à d'autres, [...] ne peut pas être maintenue et est obligée de disparaître du monde extérieur également " (Zavalloni, 2007, p. 118). Pour penser la possibilité d'une transformation du système, on doit donc s'intéresser aussi bien aux conditions par lesquelles les productions normatives se constituent qu'aux limites de ces conditions, soit l'endroit « où la reproductibilité des conditions n'est plus assurée, là où les conditions sont contingentes, transformables "(Foucault, 1976, p. 52). C'est dans une perspective proche que Moscovici décrit deux processus psychosociaux à I'œuvre dans la société qui participent ensemble au maintien et au changement de la structure sociale : la conformité et l'innovation. La conformité renvoie au fait que tous les individus, en tant qu'acteurs sociaux, utilisent et reproduisent au moins dans une certaine mesure les normes et les représentations en vigueur - ou dominantes, pourrait-on dire - en s'y conformant. Simultanément, les individus sont également capables de transformer ces représentations ou d'en proposer de nouvelles par le biais de leurs prises de position concrètes dans le champ social. Ce second processus, dit d' " innovation », implique " un conflit dont l'issue dépend autant des forces de changement que des formes de contrôle " (Moscovici, 1979, p. 14). Or, I'innovation serait un processus plus investi par les minorités sociales dans la mesure où elles ont davantage intérêt à la transformation sociale. À l'inverse, le contrôle social favorisant la conformité serait particulièrement incarné par les institutions, dont le rôle est de " transmettre des valeurs, des normes et des idéologies " (Moscovici, 1979, p. 71) et qui s'attachent à « la propagation d'une seule conception de la réalité » (Moscovici, 
1979 , p. 108) - soit la conception majoritaire. Or, pour que le contenu du champ représentationnel et normatif majoritaire puisse dans une quelconque mesure se transformer via l'influence exercée par une minorité nomique - vecteur de normes et de représentations alternatives -, un certain nombre de conditions doivent être réunies. Précisément, cette influence ne peut advenir que dans le cadre de situations sociales qui créent et alimentent l'incertitude et l'ambiguïté relativement à la stabilité du champ symbolique dominant, et qui de fait constituent un potentiel de remise en question de ce contenu symbolique (Moscovici, 1979, 2005). En ce sens, il semble que l'accès des couples de même sexe à une institution au fondement de la régulation normative de la sexualité et de la conjugalité - celle-là même qui a justifié la stigmatisation des minorités sexuelles en raison de positionnements alternatifs au modèle majoritaire - constitue une situation particulièrement privilégiée pour penser les processus et les enjeux d'un changement social potentiel. Dans cette perspective, les normes et valeurs associées aux politiques LGBTIO d'une part et celles spécifiquement portées par l'institution du mariage d'autre part apparaissent comme deux champs idéologiques que le contexte social vient mettre en dialogue, sinon en tension.

\section{VERS DES TRANSFORMATIONS SYMBOLIQUES ET SOCIÉTALES ?}

Pour ce qui concerne l'union légale en tant que telle, si l'on part du concept de conformité et que l'on se place dans une perspective réformiste, on peut considérer que la possibilité pour les couples de même sexe de se marier et l'actualisation effective de ces unions sont à même de venir renforcer la représentation selon laquelle les modalités contractuelles du mariage et les formes de conjugalité qu'il incarne constituent les formes uniques - ou pour le moins principales - par lesquelles l'intimité et la sexualité peuvent bénéficier d'une reconnaissance de la part de l'État, d'une part, et d'une légitimité aux niveaux social et symbolique d'autre part. Pour autant, on doit aussi considérer la créativité sociale inhérente à toute re-citation de la norme, puisque I'actualisation répétée de cette dernière suppose sa transformation et sa re-création permanente (Butler, 2006). Or, ce processus est d'autant plus puissant lorsque la norme est extraite de son contexte originel et que la re-citation est effectuée par un élément lui-même en marge du champ d'application habituel de cette norme, en l'occurrence les populations gaies et lesbiennes, porteuses de sexualités et d'identités jusque-là considérées comme hors norme, marginales ou alternatives relativement au champ de la sexualité normale - ou normative - encadrée par l'institution du mariage (Bem, 1993). De ce point de vue, il semble possible que l'on assiste à une transformation des modèles majoritaires depuis l'intérieur, le potentiel critique propre aux minorités concernées étant susceptible de s'actualiser - si ce n'est à travers une transformation radicale des normes de conjugalité majoritaires en tant que telles - au niveau des normes qui régulent la complémentarité entre les sexes et plus largement définissent le genre comme structure dichotomique. En effet, la reconnaissance des unions homosexuelles vient symboliquement déconstruire, ou pour le moins nuancer, l'idée d'une nécessaire complémentarité homme-femme - et par extension masculinféminin - comme condition de légitimité de l'échange amoureux ou sexuel, mais aussi 
comme principe binaire au fondement des identités individuelles. Cette reconnaissance interroge également le cadre juridique, symbolique et normatif de la filiation. En effet, si le contrat de mariage rend l'adoption possible pour les couples de même sexe, d'autres modes d'institutionnalisation de la parenté ont été mis en débat qui permettraient d'encadrer la filiation biologique pour les couples homosexuels, tels que la procréation médicalement assistée (PMA) et la gestation pour autrui (GPA). Les résistances liées à ces modes de filiation peuvent s'analyser en partie comme des manifestations de cet ordre symbolique qui définit les sexualités, filiations et unions légitimes dans la perspective de "faire famille " ainsi que les conditions de l'implication de l'État dans la procréation, en particulier au regard de la GPA. Ces résistances peuvent également se lire au regard de la symbolique de la transmission de la pathologie, fortement associée à la stigmatisation des hommes homosexuels (Joffe, 2004) et plus largement des minorités sexuelles ; on peut rappeler à dessein qu'il faut attendre les années 1990 pour assister à la déclassification de l'homosexualité comme maladie mentale par l'Organisation mondiale de la santé. Les enjeux psychosociaux liés à la filiation dans le cadre de l'ouverture du mariage aux couples de même sexe méritent une attention et un développement spécifiques qui pourraient faire l'objet de recherches futures, en particulier au regard du développement de formes de parentés pouvant venir retravailler les normes et représentations concernant les statuts et rôles parentaux traditionnels et différenciés selon le sexe des partenaires.

\section{VERS UNE TRANSFORMATION DES POLITIQUES MINORITAIRES ?}

Dans la mesure où le droit français définit depuis 2001 la vie privée non plus seulement comme protection de l'intimité, mais aussi comme un "droit à l'épanouissement » dans le champ social (Van Drooghenbroeck, 2004, p. 21), reconnaître au niveau légal les couples homosexuels selon le même cadre que les couples hétérosexuels revient à reconnaître la légitimité de leur sexualité, mais aussi par extension la légitimité de modes de vie et de normes groupales potentiellement différentes des normes majoritaires (Butler, 2006). Or, alors que le statut des populations homosexuelles - et bisexuelles - évolue via l'accession du groupe à une position sociale et légale jusque-là réservée aux membres du groupe majoritaire (en l'occurrence hétérosexuel), les populations trans et intersexes ne bénéficient en contraste d'aucune revalorisation particulière de leur statut minoritaire. Pour l'exemple, si les gays et les lesbiennes sont globalement émancipés de la dimension pathologique qui est longtemps restée attachée à la définition de l'identité homosexuelle, les personnes trans (transgenres, transsexuelles) sont toujours assujetties à un diagnostic psychiatrique dont les critères sont contestés par une partie des communautés et groupes politiques trans en particulier (Califia, 2003). Dans une perspective proche, les mouvements intersexes portent des revendications centrées sur une remise en question des protocoles médicaux actuels, et notamment des " corrections " chirurgicales et hormonales appliquées aux corps des nouveau-nés diagnostiqués comme intersexes (Fausto-

Sterling, 2000, 2012). Ces projets sociaux et politiques, cités à titre d'exemples, nous invitent à tenir compte de la diversité des contenus militants portés par les communautés LGBTIQ et de l'existence de divergences inter et intragroupes en termes de 
prises de position politiques et idéologiques, qui apparaissent comme plus ou moins radicales - ou socialement transformatrices (Savio, 2013). Par ailleurs, la critique de l'institution du mariage, ou pour le moins de son rôle hégémonique en termes de régulation des sexualités, occupe historiquement une place importante dans les cultures politiques LGBTIQ, puisque ces dernières décennies qui les ont rendues plus visibles dans le champ social sont aussi celles au cours desquelles ces politiques se sont structurées autour de leurs dimensions les plus subversives, socialement et politiquement. Ainsi, alors que la régulation de la conjugalité selon les formes dominantes proposées par le mariage (à travers les normes de monogamie, de fidélité et de filiation) pourrait être renforcée par la plus grande inclusivité de l'institution, on peut envisager que ce renforcement « menace de rendre illégitimes et abjects les arrangements sexuels [et les formes de parenté] qui ne se conforment pas à la norme du mariage sous sa forme existante ou révisée " (Butler, 2006, p. 17). Ce risque pourrait être porté dans une perspective militante par une part des communautés politiques LGBTIO et constituer, de ce fait, un nouveau contre-pouvoir relativement au régime majoritaire de régulation de la conjugalité soutenu par l'institution du mariage. En somme, si I'on considère la possibilité d'une évolution asymétrique du statut des différents groupes LGBTIO mais aussi la coexistence d'orientations différentes en termes de revendications collectives au sein de ces communautés, il est possible que des prises de position divergentes s'expriment au niveau des discours et des revendications militantes LGBTIQ là où jusqu'à présent dominait une forme d'unité, reposant sur le fait que l'ensemble de la population partageait le même statut minoritaire fondé sur une exclusion des structures sociales dominantes sinon équivalente pour les différents groupes, au moins partagée. Soulignons pour finir que si les transformations éventuelles des politiques minoritaires sont particulièrement intéressantes à considérer comme l'expression de mutations à l'œuvre dans les relations intergroupes et au niveau des dynamiques symboliques et sociétales, ces politiques sont d'ores et déjà actrices du changement social, dans la mesure où elles viennent en tant que telles questionner les structures symboliques et normatives majoritaires.

\section{CONCLUSION}

Dans le cadre de cet article, nous souhaitions mettre en lumière un certain nombre de facettes du fait social que constitue l'ouverture du mariage pour les couples de même sexe en France et son avènement légal en avril 2013. D'abord, il s'agissait de montrer que l'accès au mariage représente pour ceux et celles qui en étaient jusque-là exclus aussi bien une opportunité de valorisation du statut groupal que la « réparation » légale d'une inégalité en droit. En effet, si I'on considère les minorités sexuelles comme des groupes victimes d'une "culture de l'exclusion " (Staerklé et coll., 2007), nous sommes amenés à tenir compte des enjeux d'une oppression multiforme, constituant un contexte où le symbolique et le matériel s'entrecroisent, se chevauchent et se déterminent mutuellement. Selon une telle conception, les évolutions légales concernant les droits sociaux des groupes minoritaires sont irréductibles à une seule lecture en termes de justice sociale et prennent un sens qui dépasse la dimension strictement juridique. II s'agissait donc aussi de montrer que l'évolution de la loi est intrinsèque- 
ment liée à des enjeux idéologiques qui ont des implications en termes de changement social, via le travail des normes et des représentations et plus largement par le biais $d^{\prime}$ 'une remise en question du caractère hégémonique d'un système normatif ou d'un ordre représentationnel pour une société à un moment donné. Nous tenions enfin à mettre en évidence le fait que le changement social n'est jamais un processus unilatéral qui s'exercerait uniquement sur un champ social et symbolique majoritaire et uniforme ; au contraire, des dynamiques multiples sont à l'œuvre qui concernent l'ensemble de la société et de son capital culturel complexe - articulant des éléments en tension. En tant que contexte particulièrement représentatif, I'ouverture du mariage aux couples de même sexe nous invite en effet à penser des transformations à même de venir retravailler aussi bien les relations entre groupes majoritaires et minoritaires que le rapport de chaque groupe à sa propre position au sein de la structure sociale, relativement à ses inscriptions matérielles et symboliques spécifiques.

\section{BIBLIOGRAPHIE}

ABERLE, D. 1966. The Peyote religion among the Navaho, Chicago, Aldine.

AUGÉ, M. 1974. "Dieux et rituels ou rituels sans dieux ", dans J. Middleton (sous la direction de), Anthropologie religieuse, les dieux et les rites, Paris, Larousse, 9-36.

BEM, S.L. 1993. The Lenses of Gender : Transforming the Debate on Sexual Inequality, New Haven, CT, Yale University Press.

Bologne, J.-C. 1995. Histoire du mariage en Occident, Paris, Hachette, 2005.

BORILLO, D. 2004. "La reconnaissance juridique des couples homosexuels dans I'Union européenne : égalité ou apartheid ? ", dans V. Martens (sous la direction de), Citoyenneté, discrimination et préférence sexuelle, Bruxelles, Presses universitaires de Saint-Louis, 86-102.

BOURCIER, M.-H. 2010. "L'homosexus normaticus : Entre mariage unidimensionnel et droits sexuels ", Mouvements, 49, 1, 8-15.

BREAKWELL, G. 2010. "Resisting Representations ans Identity Processes ", Papers on Social Representations, 19, 6.1-6.11.

BUTLER, J. 2005. Trouble dans le genre : Pour un féminisme de la subversion, Paris, La Découverte.

ButLER, J. 2006. Défaire le genre, Paris, Amsterdam.

CALIFIA, P. 2003. Le mouvement transgenre : Changer de sexe, Paris, Epel.

DEMEULENAERE, P. 2003. Les normes sociales. Entre accords et désaccords, Paris, Puf.

DORLIN, E. 2008. Sexe, genre et sexualités : Introduction à la théorie féministe, Paris, Puf.

DougLAS, M. 1999. Comment pensent les institutions, Paris, La Découverte.

DUBY, G. ; PERROT, M. 1992. Histoire des femmes en Occident, Paris, Plon.

Fausto-Sterling, A. 2000. "The Five Sexes, Revisited ", Sciences (New York), $40,4$.

FAUSto-Sterling, A. 2012. Corps en tous genres. La dualité des sexes à l'épreuve de la science, Paris, La Découverte.

FELstineR, W. ; ABEL, R. ; SARAT, A. 1981. "The Emergence and Transformation of Disputes : Naming, Blaming, Claiming», Law and Society Review, 15, 630-649. 
FILLIEULE, O. 2009. «De l'objet de la définition à la définition de l'objet: De quoi traite finalement la sociologie des mouvements sociaux ? ", Politique et sociétés, 28, 1, 15-36.

FORTIN, J. 2010. L'homosexualité est-elle soluble dans le conformisme?, Paris, Textuel.

FOUCAULT, M. 1976. Histoire de la sexualité, Paris, Gallimard.

HURTIG M.-C. ; PICHEVIN, M.-F. 1986. La différence des sexes, Paris, Editions Tierce. JODELET, D. 1991. "L'idéologie dans l'étude des représentations sociales ", dans V. Aebischer, J.-P. Deconchy et E.-M. Lipiansky (sous la direction de), Idéologies et représentations sociales, Cousset (Suisse), Delval, 15-33.

JOFFE, H. 2004. "L'Autre et la construction identitaire : Entre dynamiques psychiques et dynamiques sociales ", dans M. Sanchez-Mazas et L. Licata (sous la direction de), L'Autre : regards psychosociaux, Grenoble, Presses universitaires de Grenoble, 95-116.

KASTERSZTEIN, J. 1990. "Les stratégies identitaires des acteurs sociaux : approche dynamique des finalités ", dans C. Camilleri, J. Kastersztein, E.M. Lipiansky, H. Malewska-Peyre, I. Tobaoda-Leonetti et A. Vasquez (sous la direction de), Stratégies identitaires, Paris, Puf, 28-46.

LIPIANSKY, E. M. 1991. "Représentations sociales et idéologie : analyse conceptuelle ", dans V. Aebischer, J.-P. Deconchy et E.M. Lipiansky (sous la direction de), Idéologies et représentations sociales, Cousset (Suisse), Delval, 35-63.

Moscovicl, S. 1979. Psychologie des minorités actives, Paris, Puf.

MoscoVICl, S. 1984. "The phenomenon of social representations", in R. Farr et S. Moscovici (sous la direction de), Social representations, Cambridge, Cambridge University Press, 3-69.

Moscovicl, S. 2005. "Le regard psychosocial ", Hermes, 41, 17-24.

POESCHL, G. 2003. "Inégalités sexuelles dans la mémoire collective et représentations des différences entre les sexes ", Connexions, 80, 105-124.

PREARO, N. 2014. Le moment politique de l'homosexualité : Mouvements, identités et communautés en France, Lyon, Presses universitaires de Lyon.

SAVIO, A. 2013, "Risques et stratégies identitaires pour les minorités LGBTIO. Quel(s) rapport(s) à la norme ? ", Les carnets du Greps, 5, 9-25.

StAeRKLÉ, C. ; DelAy, C. ; GiAnetTONI, L. ; RouX, P. 2007. Qui a droit à quoi ? Représentations et légitimation de l'ordre social, Grenoble, Presses universitaires de Grenoble.

TABOADA-LEONETTI, I. 1990. "Stratégies identitaires et minorités : le point de vue du sociologue ", dans C. Camilleri, J. Kastersztein, E.M. Lipiansky, H. Malewska-Peyre, I. Tobaoda-Leonetti et A. Vasquez (sous la direction de), Stratégies identitaires, Paris, Puf, 47-85.

TAJFEL, H. ; TURNER, J.C. 1979. "An integrative theory of intergrou conflict», dans S. Worchel et W. Austin (sous la direction de), The social psychology of intergroup relations, Monterey, CA, Brooks-Cole, 33-48.

TAJFEL, H. ; TURNER, J.C. 1986. "The social identity theory of intergroup behavior", dans S. Worchel et W. Austin (sous la direction de), Psychology of intergroup relations, Chicago, Nelson-Hall, 7-24.

VAN DROOGHENBROECK, S. 2004. " De la défense de l'intime au droit à l'épanouissement. Les contributions de la jurisprudence récente de la cour européenne des droits de I'homme à la cause homosexuelle ", dans V. Martens (sous la 
direction de), Citoyenneté, discrimination et préférence sexuelle, Bruxelles, Presses universitaires de Saint-Louis, 16-35.

WARNER, M. 1999. The trouble with normal: Sex, politics, and the ethics of Queer life, New York, Free Press.

ZAVALLONI, M. 2007. Égo-écologie et identité : une approche naturaliste, Paris,

Presses universitaires de France.

ANNA SAVIO, Elise Vinet et MARIE PRÉAU, DYNAMIQUeS INTERGROUPES ET CHANGEMENT SOCIAL AUTOUR DE L'OUVERTURE DU MARIAGE AUX COUPLES DE MÊME SEXE EN FRANCE

\title{
RÉSUMÉ
}

Dans le contexte français de l'ouverture du mariage aux couples de même sexe, cet article s'attache à mettre en évidence les conditions générales d'émergence et d'actualisation du changement social. À partir d'apports issus des champs de l'identité sociale et des relations intergroupes, il propose que l'ouverture à un groupe minoritaire d'une institution d'État jusque-là réservée à une part majoritaire de la population met en jeu un certain nombre de dynamiques symboliques et sociales pouvant transformer le sens et le contenu des identités sociales, minoritaires et majoritaires. En appui sur les théories s'intéressant aux conditions de transformation des normes et des représentations, I'article montre que ces dynamiques sont effectivement fondatrices du processus de changement social, dont il détaillera les enjeux pour ce contexte social et symbolique particulier.

\section{MOTS-CLÉS}

Changement social, normes, identités, union légale, minorités sexuelles.

ANNA SAVIO, Elise Vinet AND MARIE PRÉAu, INTERgROUP DYNAMICS AND SOCIAL CHANGE AROUND THE OPENING OF MARRIAGE TO SAME-SEX COUPLES IN FRANCE

\begin{abstract}
In the French context of the opening of marriage to same-sex couples, the article aims to highlight the general conditions for the emergence and actualization of social change. Going by the theoretical frameworks of social identity and intergroup relations, it suggests that the opening of a State institution to a minority group entails social dynamics and symbolic challenges which might restructure the content and meaning of the social identities involved in the process. Moreover, regarding research on the implications of - and conditions for - the transformation of social norms and representations, we are led to apprehend those social dynamics, symbolic issues and identity restructuration as significant agents of social change. On these bases and regarding the specificities of the French context, the authors also consider the possible implications of these transformations for social and symbolic fields.
\end{abstract}

KEYWORDS

Social change, norms, identities, legal union, sexual minorities. 